\title{
Multiple founder effects and geographical clustering of BRCA1 and BRCA2 families in Finland
}

\author{
Laura Sarantaus $^{1}$, Pia Huusko ${ }^{2}$, Hannaleena Eerola $^{3}$, Virpi Launonen ${ }^{2}$, Paula Vehmanen ${ }^{1}$, \\ Katrin Rapakko ${ }^{2}$, Elizabeth Gillanders ${ }^{4}$, Kirsi Syrjäkoski ${ }^{5}$, Tommi Kainu ${ }^{5}$, Pia Vahteristo ${ }^{1}$, \\ Ralf Krahe ${ }^{6}$, Kati Pääkkönen ${ }^{6}$, Jaana Hartikainen ${ }^{7}$, Carl Blomqvist ${ }^{3}$, Tuija Löppönen ${ }^{2}$, \\ Kaija Holli ${ }^{8}$, Markku Ryynänen ${ }^{7}$, Ralf Bützow ${ }^{1}$, Åke Borg ${ }^{9}$, Brita Wasteson Arver ${ }^{10}$, \\ Eva Holmberg ${ }^{11}$, Arto Mannermaa ${ }^{7}$, Juha Kere ${ }^{12}$, Olli-Pekka Kallioniemi ${ }^{5}$, \\ Robert Winqvist ${ }^{2}$ and Heli Nevanlinna ${ }^{1}$
}

\begin{abstract}
${ }^{1}$ Department of Obstetrics and Gynecology, and ${ }^{3}$ Department of Oncology, Helsinki University Central Hospital; ${ }^{2}$ Department of Clinical Genetics, University of Oulu and Oulu University Hospital, Finland; ${ }^{4} \mathrm{Cancer}$ Genetics Branch, National Human Genome Research Institute, National Institutes of Health, Bethesda, MD, USA; ${ }^{5}$ Laboratory of Cancer Genetics, Institute of M edical Technology, and ${ }^{8}$ Department of Oncology, Tampere University Hospital; ${ }^{6}$ Department of Medical Genetics, and ${ }^{12}$ Finnish Genome Centre, University of Helsinki; ${ }^{7}$ Unit of Clinical Genetics of the Department of Gynecology, Kuopio University Hospital and University of Kuopio, Finland;

${ }^{9}$ Department of Oncology, University Hospital, Lund; ${ }^{10}$ Department of Clinical Genetics and Institution of M ol ecular Medicine, CMM2, Karolinska Hospital, Stockholm; ${ }^{11}$ Department of Clinical Genetics, Norrland University Hospital, Umeå, Sweden
\end{abstract}

In the Finnish breast and ovarian cancer families six BRCA1 and five BRCA2 mutations have been found recurrently. Some of these recurrent mutations have also been seen elsewhere in the world, while others are exclusively of Finnish origin. A haplotype analysis of 26 Finnish families carrying a BRCA1 mutation and 20 families with a BRCA2 mutation indicated that the carriers of each recurrent mutation have common ancestors. The common ancestors were estimated to trace back to 7-36 generations (150-800 years). The time estimates and the geographical clustering of these founder mutations in Finland are in concordance with the population history of this country. Analysis of the cancer phenotypes showed differential ovarian cancer expression in families carrying mutations in the $5^{\prime}$ and $3^{\prime}$ ends of the BRCAl gene, and earlier age of ovarian cancer onset in families with BRCA1 mutations compared with families with BRCA2 mutations. The identification of prominent and regional BRCA1 and BRCA2 founder mutations in Finland will have significant impact on diagnostics in Finnish breast and ovarian cancer families. An isolated population with known history and multiple local founder effects in multigenic disease may offer distinct advantages also for mapping novel predisposing genes. European Journal of Human Genetics (2000) 8, 757-763.

Keywords: BRCA1; BRCA2; haplotype; founder mutations; breast cancer; ovarian cancer

Correspondence: Dr Heli Nevanlinna, Department of Obstetrics and Gynecology, Helsinki University Central Hospital, Haartmaninkatu 2, FIN-00029 HUS, Finland. Tel: +358947172841; Fax: +358947172973; E-mail: heli.nevanlinna@hus.fi, and Dr Robert Winqvist, Department of Clinical Genetics, Oulu University Hospital, Kajaanintie 50,

FIN-90220 Oulu, Finland. Tel: + 35883153228 ; Fax: +35883153243;

E-mail: robert.winqvist@oulu.fi

Received 28 January 2000; revised 29 May 2000; accepted 6 June 2000 


\section{Introduction}

The cloning of $\mathrm{BRCA} 1^{1}$ and $\mathrm{BRCA} 2,{ }^{2}$ the major known genes predisposing to early-onset breast cancer and ovarian cancer, has resulted in rapid identification of a large number of families with mutations in these genes. ${ }^{3}$ Although both genes exhibit numerous distinct mutations, several al terations have been found recurrently in defined ethnic and geographic populations, especially in the Ashkenazim and in Iceland. ${ }^{4-6}$ In Finland, 11 distinct BRCA1 and seven BRCA2 mutations have been detected, ${ }^{7-9}$ and six of the BRCA1 and five of the BRCA2 mutations are recurrent. Some of these mutations are unique to Finland, while others have also been reported elsewhere in the world, like BRCA2 999del5, a strong founder mutation in Iceland as well. ${ }^{4,6}$

We have here investigated the mutation-associated haplotype conservation in Finnish BRCA1 and BRCA2 families. We have estimated the time since the common ancestor for the families segregating the same mutation and also studied the geographical distribution of the alterations in Finland. In addition, the breast and ovarian cancer phenotypes of the families were analysed.

\section{Material and methods \\ Families studied}

Altogether 26Finnish breast or breast-ovarian cancer families carrying recurrent BRCA 1 mutations and 20 families with
BRCA2 mutations were studied for haplotype conservation, and 34 BRCA1 and 37BRCA2 families for the cancer phenotype. The families had previously been ascertained and mutations identified ${ }^{7-9}$ in four University Hospitals in Finland (Helsinki, Oulu, Tampere, and Kuopio, additional unpublished data). The permission for the genetic analysis was obtained by written informed consent. The family data were collected by interviewing the index patients as well as by searching church parish records and population registry data. Cancer diagnoses and ages at onset were obtained from hospital records, death certificates or family questionnaires. The diagnoses were confirmed through the Finnish Cancer Registry whenever possible. The study was approved by ethical committees of the hospitals and appropriate permissions were obtained from the Ministry of Social Affairs and Health in Finland. For comparison of the mutation-associated haplotypes of the BRCA1 3744delT mutation in Finland and Sweden, ${ }^{10}$ samples from four Swedish families were also included.

\section{Mutations studied}

The BRCA1 and BRCA2 mutations identified in Finland and the recurrent BRCA1 and BRCA2 mutations studied in the haplotype analysis are indicated in Table1. Mutation-specific breast and ovarian cancer phenotypes are also shown in Table1.

Table 1 Mutations and phenotypes of the BRCA1 and BRCA2 families in Finland

\begin{tabular}{|c|c|c|c|c|}
\hline Gene and mutation & $\begin{array}{l}\text { No. of } \\
\text { families in } \\
\text { Finland }\end{array}$ & 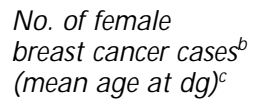 & $\begin{array}{l}\text { No. of ovarian } \\
\text { cancer cases }^{b} \\
(\text { mean age at } \mathrm{dg})^{c}\end{array}$ & Reported outside Finland \\
\hline \multicolumn{5}{|l|}{ BRCA1 } \\
\hline Ex 11, 1924delA & 1 & $1(44)$ & - & No \\
\hline Ex 11,2803 delAA & 2 & $3(56)$ & $2(62)$ & the Netherlands ${ }^{30}$ \\
\hline Ex 11,3604 del $A^{a}$ & 6 & $7(45)$ & $10(46)$ & Belgium, the Netherlands ${ }^{30}$ \\
\hline Ex 11,3744 delT $^{a}$ & 8 & $7(45)$ & $10(49)$ & Sweden 10 \\
\hline Ex 11, 4153delA & 1 & $1(32)$ & $1(48)$ & Latvia, Poland, Russia, Sweden ${ }^{3,28,37}$ \\
\hline Int $11,4216 n t-2 A \rightarrow G^{a}$ & 9 & $24(43)$ & $8(52)$ & No \\
\hline $\mathrm{Ex} 13,4446 \mathrm{C} \rightarrow \mathrm{T}^{\mathrm{a}}$ & 3 & $23(46)$ & $8(53)$ & Belgium, Canada, France, UK, USA $3,28,29$ \\
\hline Ex 17,5145 del11 & 1 & $4(37)$ & - & No \\
\hline Ex $20,5370 C \rightarrow T^{a}$ & 3 & $12(49)$ & $3(67)$ & Austria 31 \\
\hline Ex 20,5382 insC & 1 & $2(57)$ & $1(40)$ & $\begin{array}{l}\text { Austria, Belgium, Canada, France, Germany, Hungary, } \\
\text { Israel, Italy, Latvia, the Netherlands, Russia, UK, } \\
\text { USA }^{3,28,37}\end{array}$ \\
\hline \multicolumn{5}{|l|}{ BRCA2 } \\
\hline Ex 9, 999del5 & 13 & $52(47)$ & $6(60)$ & Iceland 6 \\
\hline Ex 11,4081 insA & 1 & $2(67)$ & $2(60)$ & No \\
\hline Ex $11,6495 / 6496 G \rightarrow C$, delCA & 1 & $3(52)$ & - & No \\
\hline Ex 11, 6503delTT ${ }^{a}$ & 3 & $8(57)$ & $4(62)$ & Belgium, the Netherlands, Sweden, UK, USA ${ }^{32,33}$ \\
\hline Ex $15,7708 C \rightarrow T^{a}$ & 8 & $26(45)$ & $4(56)$ & No \\
\hline Ex $18,8555 \mathrm{~T} \rightarrow \mathrm{G}^{\mathrm{a}}$ & 5 & $12(49)$ & $1(60)$ & No \\
\hline Int $23,9346 n t-2 A \rightarrow G^{a}$ & 9 & $20(52)$ & $4(66)$ & No \\
\hline Total & 40 & $123(48)$ & 21 (61) & \\
\hline
\end{tabular}

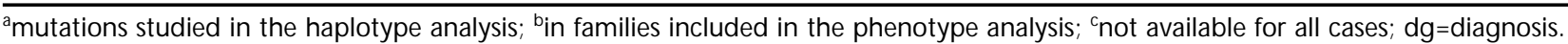




\section{Genotyping}

Eleven polymorphic microsatellite markers spanning $26 \mathrm{cM}$ around $\mathrm{BRCA}^{11-15}$ on chromosome17q21 and 17 polymorphic microsatellite markers spanning $36 \mathrm{cM}$ around $\mathrm{BRCA} 2^{16-18}$ on chromosome $13 \mathrm{q} 12$ were used for genotyping (Figure1). Primer sequences for markers D13S260 through D13S267 were positioned and marker order and physical distances were determined using Sequencher v3.0 (Gene Codes Corporation, Ann Arbor, MI, USA) and the genomic sequence $^{19}$ for this region. Physical distances for markers D13S260 through D13S267 were converted to centiMorgans

$\begin{array}{ll}\begin{array}{l}\text { a } \\ \text { CM }\end{array} & \begin{array}{l}\text { Marker } \\ \text { Cen }\end{array} \\ 3 & \text { D17S1872 } \\ 3 & \text { D17S946 } \\ 2 & \text { D17S250 } \\ 0.7 & \text { THRA1 } \\ 0.6 & \text { D17S800 } \\ 1.2 & \text { D17S846 } \\ 0.4 & \text { BRCA1 D17S855 } \\ 1.1 & \text { D17S902 } \\ 6 & \text { D17S579 } \\ 8 & \text { D17S588 } \\ & \text { D17S787 } \\ & \text { tel }\end{array}$

tel

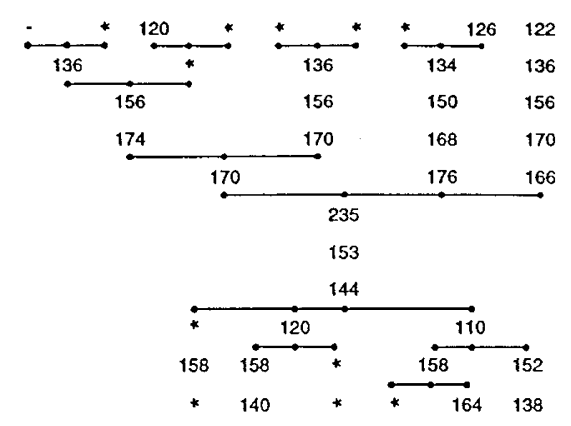

b

cM Marker

cen

D135283

D13S1294

D135217

D13S1246

D13S260

D13S1699

D1351698

D13S1697

D135171

D13S1695

D13S1694

D13S1696

D13S267

D13S263

D13S1227

D13S1272

D13S153

tel

Figure 1 A haplotype reconstruction for a BRCA1 3744delT and for b BRCA2 9346nt-2A $\rightarrow \mathrm{G}$. The order of markers studied and their relative distances in $\mathrm{CM}$ are on the left; the historical recombinations are on the right. Alleles are designated according to their size in base pairs $*=$ ambiguous allele; - = unknown allele. assuming $1 \mathrm{cM}=0.5 \mathrm{Mb}$, which was the average observed ratio at this region.

For 17q21 markers PCR products were labelled with $\left[\alpha^{32} \mathrm{P}\right]-d C T P$ and separated by denaturing polyacrylamide gel (7\%) electrophoresis. Biomax X-ray films (Kodak, NY, NY, USA) were used for autoradiography. Allele sizes were determined using M13mp18 marker (Sequenase Kit, USB, Cleveland, $\mathrm{OH}, \mathrm{USA})$.

For $13 q 12$ markers forward PCR primers were fluorescent dye labelled with either FAM, HEX or TET (PE Applied Biosystems, Foster City, CA, USA). The amplification products were separated on an ABI 377 instrument (PE Applied Biosystems), and genotype data were analysed using GeneScan 3.1 and Genotyper 2.0 software from PE Applied Biosystems. Allele sizes were matched according to the genotype of CEPH reference individual 134702 (PE Applied Biosystems).

In order to define the allele frequencies in the Finnish population, 42 and 96 healthy individuals were genotyped for chromosome17q21 and 13q12 markers, respectively.

\section{Haplotyping and estimation of the time since common ancestors (spreading of the mutations)}

The haplotypes were constructed by Genehunter program and/or manually. Historical recombinations were reconstructed assuming minimal parsimony. Starting from the site of the mutation and moving outwards in both directions, historical recombinations were noted as the branching of the haplotype when two or more different alleles were observed for a marker. Examples of such recombinational histories for the BRCA1 3744delT and BRCA2 9346nt-2A $\rightarrow$ G chromosomes are depicted in Figure 1.

The number of generations $(\mathrm{g})$ since the common ancestor of the families studied, denoted in the following as the time since spreading of the mutation, was estimated by the LuriaDelbrück equation ${ }^{20}$ of $p_{\text {excess }}=\alpha(1-\theta)^{g}$, where $\alpha=1$ (all chromosomes carry the same mutation), $\theta$ refers to the recombination fraction between the mutation and marker locus, and $p_{\text {excess }}=\left(p_{\text {affected }}-p_{\text {normal }}\right) /\left(1-p_{\text {normal }}\right)$, where $p_{\text {affec- }}$ ted $=$ fraction of the ancestral chromosomes (allele) of all affected chromosomes and $p_{\text {normal }}=$ frequency of the same allele in normal population chromosomes. The average of the estimations was considered as the most likely time since the common ancestor. Alternatively, a modification was applied where $p_{\text {excess }}$ value for each marker was the minimum and maximum fraction of chromosomes carrying the most common shared allele for that marker based on the branched haplotype tree.

\section{Phenotype analysis}

Thirty-four families with a BRCA1 and 37 with a BRCA2 mutation were examined for breast and ovarian cancer phenotype. All cancer cases in the families were included in the phenotype study, with the exception of those who were known not to be BRCA1 or BRCA2 mutation carriers. 
Correlation between the position of the mutation ( $5^{\prime}$ versus $3^{\prime}$ end of the gene) and the breast and ovarian cancer phenotype in the BRCA 1 families was analysed using Fisher's exact test. Only one of the BRCA2 mutations (6503delTT) is located in the ovarian cancer cluster region (OCCR), ${ }^{21}$ which does not allow assessment of the genotype-phenotype correlation. The variation of the age at diagnosis was analysed for both genes and both phenotypes by unpaired t-test. Cumulative age-specific percentages of age at onset for breast and ovarian cancer using 5-year intervals were determined for the BRCA1 and BRCA2 families as well as the general population. $^{22}$

\section{Results and discussion \\ Haplotype analysis}

The haplotype analysis showed that the carriers of each recurrent mutation have common ancestors. Variation in the length of the shared haplotype indicates that distinct mutations probably started to spread at different time periods, which is also supported by the geographical distribution of the mutations and their relationship to the population history and settlement of the country. The conserved core haplotypes as well as the time estimates and geographical distribution of the origins of the families are shown in Figure2. Both methods used for estimating the time since the spread of the mutations began gave consistent results. The estimates derived from using the population-based allele frequencies were within the range of minimum and maximum values derived from the modification using information from the branched haplotype tree.

Recently, recombination suppression in a region of $200-400 \mathrm{~kb}$ including and immediately adjacent to BRCA1 has been reported. ${ }^{23}$ The Luria-Del brück calculation uses data from observed historically recombined chromosomes. The generation estimates are based on the average value of all recombined markers studied and the common haplotypes extended outside the suppression region. Thus the short suppressed region that does not recombine essentially looks like a 'point' in the genome and is expected to have little effect on the time estimates here. Interestingly, more extensive variation of the alleles was denoted in this study than reported by Liu and Barker. ${ }^{23}$ Among the five different BRCA1 mutation haplotypes, four different alleles were present in the intragenic marker D17S855 within the reported recombination suppression region.

The BRCA1 mutation 3744delT (haplotype reconstruction shown in Figure 1a) was estimated to have started to spread 23-36generations (500-700years) ago. The alleles in the short conserved haplotype ( $1.6 \mathrm{cM})$ of the seven Finnish and four Swedish families analysed are rare (estimated haplotype frequency in the Finnish population $0.57 \%$ ), supporting the common origin of the families. According to the church records, a majority of the Finnish families have lived in Central Ostrobothnia for at least 300 years, whereas the
Swedish ones come from the opposite side of the Gulf of Bothnia. Thus, the mutation could have been brought across the sea from Sweden to Finland, along with Swedish settlers. $^{24}$

The origins of the families with the $4216 n t-2 A \rightarrow G$ mutation also cluster in Central Ostrobothnia and further in Central Finland inhabited after the fifteenth century, during the more permanent settlement of central and northern parts of the country. ${ }^{24,25}$ This mutation is unique to the Finns, and although it is the most frequent BRCA1 mutation in Finland, we estimated that the spread of this mutation started fairly recently (less than 10 generations ago).

The $4446 \mathrm{C} \rightarrow \mathrm{T}$ mutation is reported frequently in the Breast Cancer Information Core database (BIC), ${ }^{3}$ and has been detected at least in Belgium, Canada, France, the UK and the USA. Several distinct disease-linked haplotypes segregating with this mutation indicate independent origins of the same mutation. ${ }^{26-29}$ In Finland, the three kindreds share a $7.9 \mathrm{cM}$ core haplotype and the origins of the families cluster in a remote geographical location in Southern Karelia, near the Russian border. This particular mutation could be a distinct mutational event in Finland, supporting the hypothesis of a hotspot site. The 3604del A has also been reported several times in $\mathrm{BIC}^{3}$ and has previously been found in Belgium and the Netherlands. ${ }^{30}$ The geographical location of all the families in the most southern and coastal part of Finland may suggest that the mutation originated from Central Europe. Besides Finland, 5370 C $\rightarrow$ T has been identified in Austria. ${ }^{31}$

The BRCA2 mutation 9346nt-2A $\rightarrow \mathrm{G}$ (haplotype reconstruction shown in Figure $1 b$ ) has been found in nine different families, and represents an alteration unique to the Finns. The spread of this mutation was estimated to have started 7-11generations (150-200years) ago. This is also supported by the distribution of the origins of the families in the northern and eastern parts of the country that were settled after the fifteenth century, followed by regional population expansions in the seventeenth century. ${ }^{24}$

The $7708 \mathrm{C} \rightarrow \mathrm{T}$ mutation, which is also unique to the Finns, seems to be of older origin (200-400years) with dispersed geographical pattern both in the regions of older as well as more recent inhabitation. The $8555 \mathrm{~T} \rightarrow \mathrm{G}$ mutation is also unique to Finland, and all the families originate from Pirkanmaa. Although this part of the country was inhabited early in our population history, this mutation is not widespread but has remained as a regional founder mutation for 7-9 generations.

The geographical distribution of 6503delTT is quite similar to that of $9346 \mathrm{nt}-2 \mathrm{~A} \rightarrow \mathrm{G}$ as both mutations have spread into the late settlement area. 6503delTT has been listed several times in $\mathrm{BIC}^{3}$ and families with this mutation have been reported in Belgium, the Netherlands, Sweden, the UK, and the USA. 3,32,33 The conserved haplotype of the Finnish families includes completely different allele sizes from those 
reported elsewhere, indicating distinct origins of the 6503delTT mutation. ${ }^{33}$

Phenotypic studies have suggested that BRCA1 mutations in families with a high proportion of ovarian cancer tend to be located at the $5^{\prime}$ end, whilst families exhibiting predominantly breast cancer seem to have mutations at the $3^{\prime}$ end of the gene. ${ }^{34} \mathrm{~A}$ significant correlation between the location of the BRCA1 mutation and the cancer phenotype was also
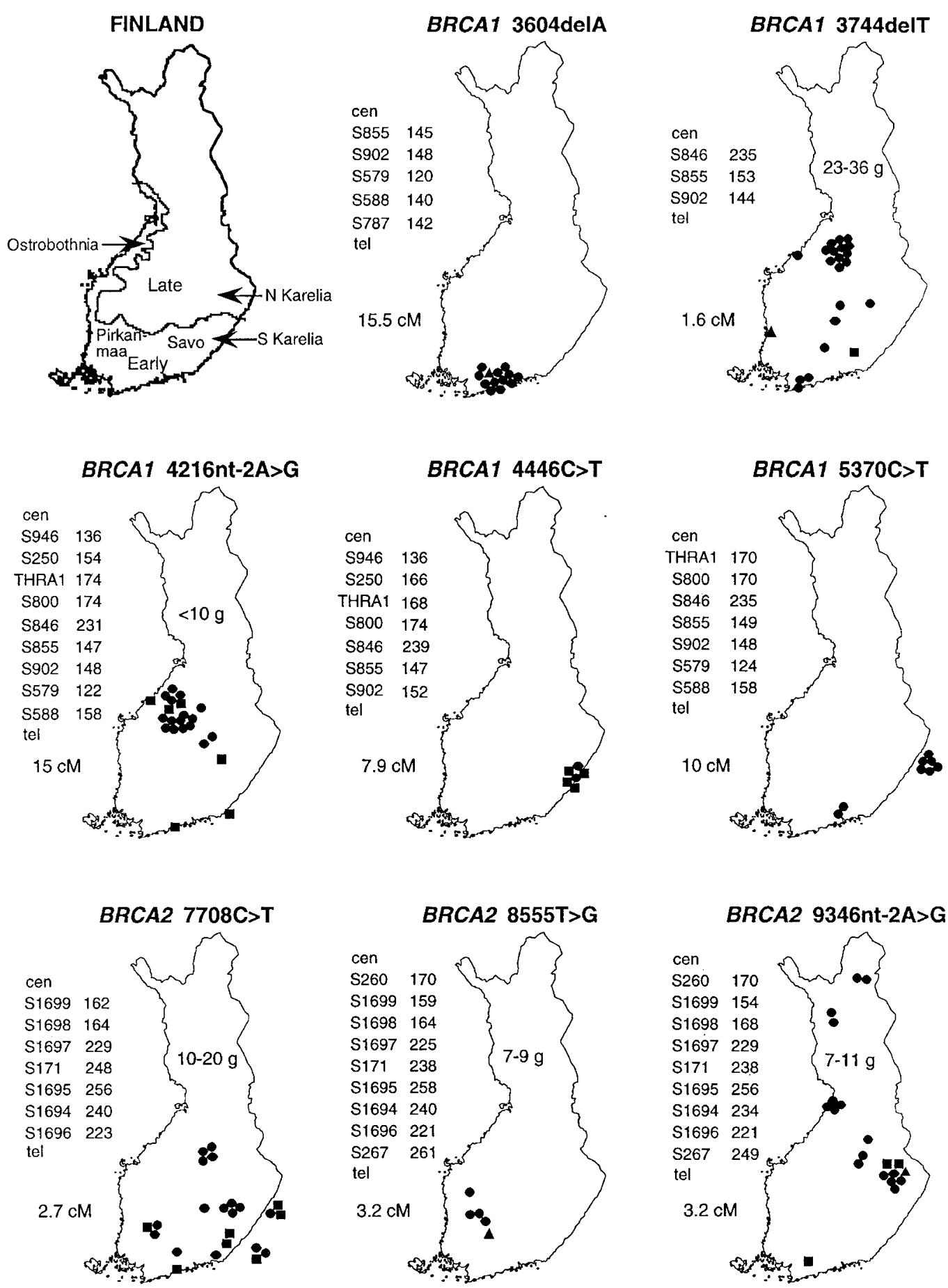

Figure 2 Maps of Finland showing early and late settlement areas and geographical distribution of the ancestry of recurrent BRCA1 and BRCA2 mutations. Birthplaces of grandparents, when known, are marked by black circles. Otherwise, birthplaces of parents (black squares) or index persons (black triangles) are shown. The conserved core haplotypes (alleles designated according to their size in base pairs) and the estimations of the number of generations $(\mathrm{g})$ since the common ancestor are indicated. 
a

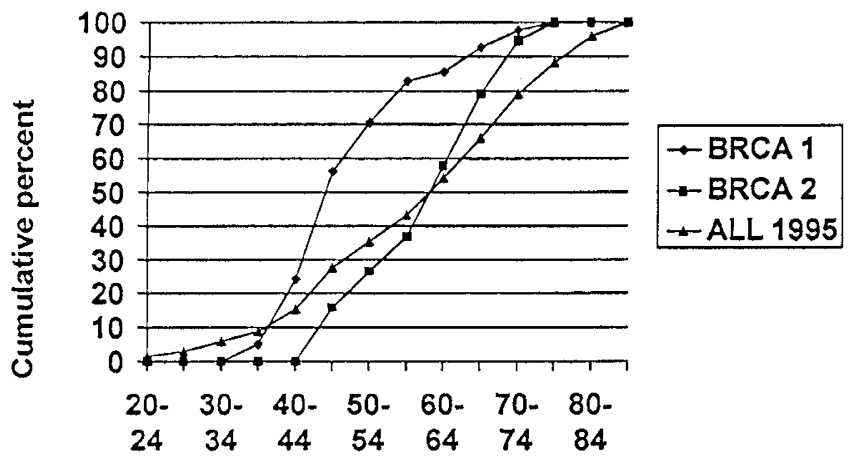

b

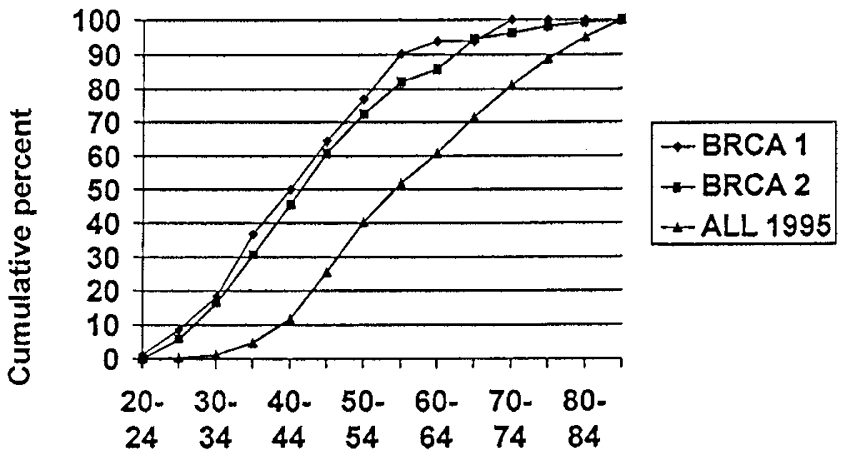

Figure 3 a Cumulative age-specific percentages of age at onset for ovarian cancer and b breast cancer in BRCA1 and BRCA2 families. All 1995 means all ovarian a and breast b cancer cases diagnosed in Finland in the year $1995 .^{22}$

found here. The proportion of ovarian cancer was significantly higher ( $P=0.001$, Fisher's exact test), with a 2.3-fold difference, in families carrying mutations in exon 11 compared to the families with mutations $3^{\prime}$ of this exon. Altogether, phenotype information was available from 34 BRCA 1 families and 37 BRCA 2 families, and ovarian cancer was present in $74 \%$ and $43 \%$, respectively (Table1). The age at ovarian cancer onset was significantly earlier in the BRCA1 families (mean 51 years) than in the BRCA2 families (mean 61 years) ( $P<0.0005$, unpaired t-test), with almost $60 \%$ of the BRCA1 cases diagnosed before age 50 (Figure3a). However, the distribution of diagnostic ages for breast cancer was similar in the BRCA1 (mean 46years) and BRCA2 (mean 48 years) families (Figure 3b). Identification of larger number of individuals with these prevalent founder mutations in Finland will facilitate analysis of mutation-specific cancer risks and phenotypes. This will affect the clinical management of families carrying these mutations. Furthermore, environmental or other genetic factors modifying the cancer risks can ideally be studied in a genetically homogeneous population, especially in families carrying identical mutations.

In Finland the 11 known recurrent BRCA1/2 mutations cover $84 \%$ of all BRCA1 and BRCA2 families found in screening the entire genes. ${ }^{7-9}$ Whilst an unknown fraction, perhaps as high as almost $40 \%$ of mutations, may remain undetected by any mutation detection method, ${ }^{35}$ linkage analysis in 24 of our large mutation-negative families suggested possible linkage to either BRCA1 or BRCA2 in only 4 (17\%) (Kainu T et al, 2000, unpublished data). Thus, the major Finnish founder mutations may already have been identified. The high coverage of the founder mutations in Finland has also significant impact on diagnostics, which can first be based on direct screening of these mutations. Especially in some parts of the country, the mutation spectrum is very narrow and specific, whereas in the capital region of Helsinki, almost all Finnish mutations have been detected. Finally, the observation of multiple local founder effects in a multigenic disease provides support for the concept that an isolated population with known history may offer distinct advantages also for mapping novel predisposing genes. ${ }^{36}$

\section{Acknowledgements}

The authors wish to thank The Finnish Cancer Registry for cancer data. The help and advice of Drs Guillermo Blanco, Jaakko Leisti, Annika Lindblom, Susan Neuhausen and Ulla Puistola is gratefully acknowledged. We also wish to thank nurses Minna Merikivi and Leena Kukkola for handling patient contacts and Marika Kujala for technical assistance. We are greatly indebted to all patients and their family members for volunteering to participate in these studies. This study was supported by the Helsinki University Central Hospital Research Funds, the University of Oulu, the Oulu University Hospital, the Research Fund of Tampere University Hospital, Academy of Finland, the Nordic Cancer Union, the Finnish Cancer Society, the Cancer Foundation of Northern Finland, the Pirkanmaa Cancer Society, the Ida Montin Foundation, the Foundation of Ella and Georg Ehrnrooth, and the Finnish Breast Cancer Group. Laura Sarantaus and Pia Huusko contributed equally to this study.

\section{References}

1 Miki Y, Swensen J, Shattuck-Eidens D et al: A strong candidate for the breast and ovarian cancer susceptibility gene BRCA1. Science 1994; 266: 66-71.

2 Wooster R, Bignell G, Lancaster J et al: Identification of the breast cancer susceptibility gene BRCA2. Nature 1995; 378: 789-792.

3 Breast Cancer Information Core database (BIC): http://www .nhgri.nih.gov/Intramural research/Lab transfer/Bic/

4 Gudmundsson J, Johannesdottir G, Arason A et al: Frequent occurrence of BRCA2 linkage in Icelandic breast cancer families and segregation of a common BRCA2 haplotype. Am J Hum Genet 1996; 58: 749-756.

5 Roa BB, Boyd AA, Volcik K, Richards CS: Ashkenazi Jewish population frequencies for common mutations in BRCA1 and BRCA2. Nat Genet 1996; 14: 185-187. 
6 Thorlacius S, Olafsdottir G, Tryggvadottir L et al: A single BRCA2 mutation in male and female breast cancer families from Iceland with varied cancer phenotypes. Nat Genet 1996; 13: 117-119.

7 Vehmanen P, Friedman LS, Eerola $\mathrm{H}$ et al: Low proportion of BRCA1 and BRCA2 mutations in Finnish breast cancer families: evidence for additional susceptibility genes. Hum Mol Genet 1997; 6: 2309-2315.

8 Vehmanen $\mathrm{P}$, Friedman LS, Eerola $\mathrm{H}$ et al: A low proportion of BRCA2 mutations in Finnish breast cancer families. Am J Hum Genet 1997; 60: 1050-1058.

9 Huusko P, Pääkkönen $K$, Launonen $V$ et al: Evidence of founder mutations in Finnish BRCA1 and BRCA2 families. Am J Hum Genet 1998; 62: 1544-1548.

10 Zelada-Hedman M, Wasteson Arver B, Claro A et al: A screening for BRCA1 mutations in breast and breast-ovarian cancer famlies from the Stockholm region. Cancer Res 1997; 56: 2474-2477.

11 Feunteun J, Narod SA, Lynch HT et al: A breast-ovarian cancer susceptibility gene maps to chromosome17q21. Am J Hum Genet 1993; 52: 736-742.

12 Albertsen HM, Smith SA, Mazoyer S et al: A physical map and candidate genes in the BRCA1 region on chromosome17q12-21. Nat Genet 1994; 7: 472-479.

13 Murrell JR, Koller D, Foroud T et al: Familial multiple-system tauopathy with presenile dementia is localized to chromosome17. Am J Hum Genet 1997; 61: 1131-1138.

14 National Center for Biotechnology Information: http://www ncbi.nlm.nih.gov/

15 Genome Database: http://gdbwww.gdb.org/

16 Dib C, Faure S, Fizames C et al: A comprehensive genetic map of the human genome based on 5,264 microsatellites. Nature 1996; 380: 152-154.

17 Couch FJ, Rommens JM, Neuhausen SL et al: Generation of an integrated transcription map of the BRCA2 region on chromosome13q12-q13. Genomics 1996; 36: 86-99.

18 Généthon: ftp://ftp.genethon.fr/pub/Gmap/Nature-1995/

19 The Sanger Centre: ftp://ftp.sanger.ac.uk/pub/human/sequences/ Chr 13/

20 Lehesjoki A-E, Koskiniemi M, Norio R et al: Localization of the EPM 1 gene for progressive myoclonus epilepsy on chromosome21: linkage disequilibrium allows high resolution mapping. Hum Mol Genet 1993; 2: 1229-1234.

21 Gayther SA, Mangion J, Russell P et al: Variation of risks of breast and ovarian cancer associated with different germline mutations of the BRCA2 gene. Nat Genet 1997; 15: 103-105.

22 Finnish Cancer Registry - Institute for Statistical and Epidemiological Cancer Research. Cancer insidence in Finland 1995: Cancer Statistics of the National Research and Development Centre for W elfare and Health. Cancer Society of Finland: Publication No.58, Helsinki, 1997.
23 Liu X, Barker DF: Evidence for effective suppression of recombination in the chromosome17q21 segment spanning RNU2-BRCA1. Am J Hum Genet 1999; 64: 1427-1439.

24 Norio R, Nevanlinna HR, Perheentupa J: Hereditary diseases in Finland; rare flora in rare soil. Ann Clin Res 1973; 5: 109-141.

25 Nevanlinna HR: The Finnish population structure. A genetic and genealogical study. Hereditas 1972; 71: 195-236.

26 Neuhausen SL, Mazoyer S, Friedman L et al: Haplotype and phenotype analysis of six recurrent BRCA1 mutations in 61 families: results of an international study. Am J Hum Genet 1996; 58: 271-280.

27 Stoppa-Lyonnet D, Laurent-Puig P, Essioux L et al: BRCA1 sequence variations in 160 individuals referred to a breast/ovarian family cancer clinic. Am J Hum Genet 1997; 60: 1021-1030.

28 Szabo $\mathrm{Cl}$, King M-C: Population genetics of BRCA1 and BRCA2. Am J Hum Genet 1997; 60: 1013-1020.

29 Tonin PN, Mes-Masson A-M, Futreal PA et al: Founder BRCA1 and BRCA2 mutations in French Canadian breast and ovarian cancer families. Am J Hum Genet 1998; 63: 1341-1351.

30 Peelen $T$, van Vliet $M$, Petrij-Bosch $A$ et al: A high proportion of novel mutations in BRCA1 with strong founder effects among Dutch and Belgian hereditary breast and ovarian cancer families. Am J Hum Genet 1997; 60: 1041-1049.

31 Wagner TMU, Möslinger RA, Muhr D et al: BRCA1-related breast cancer in Austrian breast and ovarian cancer families: specific BRCA 1 mutations and pathological characteristics. Int J Cancer 1998; 77: 354-360.

32 Mazoyer S, Dunning AM, Serova $O$ et al: A polymorphic stop codon in BRCA2. Nat Genet 1996; 14: 253-254.

33 Neuhausen SL, Godwin AK, Gershoni-Baruch R et al: Haplotype and phenotype analysis of nine recurrent BRCA2 mutations in 111 families: results of an international study. Am J Hum Genet 1998; 62: 1381-1388.

34 Gayther SA, Warren W, Mazoyer Set al: Germline mutations of the BRCA1 gene in breast and ovarian cancer families provide evidence for a genotype-phenotype correlation. Nat Genet 1995; 11: 428-433.

35 Ford D, Easton DF, Stratton $M$ et al: Genetic heterogeneity and penetrance analysis of the BRCA1 and BRCA2 genes in breast cancer families. Am J Hum Genet 1998; 62: 676-689.

36 Wright $A F$, Carothers $A D$, Pirastu $M$ : Population choice in mapping genes for complex diseases. Nat Genet 1999; 23. 397-404.

37 Csokay B, Tihomirova L, Stengrevics A, Sinicka O, Olah E: Strong founder effects in BRCA1 mutation carrier breast cancer patients from Latvia. Mutation in brief no. 258. Online. Hum Mutat 1999; 14: 92. 